\title{
High-Speed 3-D Electroholographic Movie Playback Using a Digital Micromirror Device
}

\author{
Naoki TAKADA $^{\dagger a)}$, Member, Masato FUJIWARA ${ }^{\dagger \dagger}$, ChunWei OOI ${ }^{\dagger \dagger \dagger}$, Yuki MAEDA ${ }^{\dagger \dagger}$, Nonmembers, \\ Hirotaka NAKAYAMA ${ }^{\dagger \dagger \dagger \dagger}$, Member, Takashi KAKUE ${ }^{\dagger \dagger \dagger \dagger}$, Nonmember, Tomoyoshi SHIMOBABA ${ }^{\dagger \dagger \dagger \dagger}$, \\ and Tomoyoshi ITO ${ }^{\dagger \dagger \dagger \dagger}$, Members
}

\begin{abstract}
SUMMARY This study involves proposing a high-speed computergenerated hologram playback by using a digital micromirror device for high-definition spatiotemporal division multiplexing electroholography. Consequently, the results indicated that the study successfully reconstructed a high-definition 3-D movie of 3-D objects that was comprised of approximately 900,000 points at $60 \mathrm{fps}$ when each frame was divided into twelve parts.

key words: electroholography, spatiotemporal division multiplexing method, digital micromirror device, computer-generated hologram
\end{abstract}

\section{Introduction}

Holography [1] corresponds to an extremely promising 3-D technology because it is used to directly record and faithfully reconstruct a 3-D image. Electroholography using computer-generated holograms (CGHs) [2] is considered as an ultimate 3-D television (TV) [3]. An aerial projection of 3-D movies using electroholography [4] and a table screen 360-deg holographic display [5], [6] ware reported.

However, a 3-D TV using electroholography requires high-performance computational power to reconstruct 3-D images from CGHs in real time. In order to overcome this problem, fast CGH calculation techniques have been reported [7]-[14]. In recent years, graphics processing units (GPUs) exhibit high computational performance at low cost. Thus, electroholography using GPUs was actively investigated by extant studies [15]-[27].

However, the display of high-definition CGH obtained for 3-D objects that are comprised of huge numbers of object points requires a wide dynamic range that cannot be achieved with the present spatial light modulator (SLM). Thus, currently available SLMs are unable to faithfully display the CGH. Therefore, the image quality of the ob-

Manuscript received February 28, 2017.

Manuscript revised June 2, 2017.

${ }^{\dagger}$ The author is with the Science Department, Natural Sciences Cluster, Research and Education Faculty, Kochi University, Kochishi, 780-8520 Japan.

${ }^{\dagger \dagger}$ The authors are with the Graduate School of Integrated Arts and Sciences, Kochi University, Kochi-shi, 780-8520 Japan.

${ }^{\dagger \dagger \dagger}$ The author is with the Faculty of Science, Kochi University, Kochi-shi, 780-8520 Japan.

${ }^{\dagger+\dagger}$ The author is with the Center for Computational Astrophysics, National Astronomical Observatory of Japan, Mitaka-shi, 181-8588 Japan.

${ }^{\dagger+1+\dagger}$ The authors are with the Graduate School of Engineering, Chiba University, Chiba-shi, 263-8522 Japan.

a)E-mail: ntakada@is.kochi-u.ac.jp

DOI: $10.1587 /$ transele.E100.C.978 tained CGH deteriorates when the $\mathrm{CGH}$ is displayed on an SLM [28].

Furthermore, speckle noise is generated from unwanted interference and periodic location of the reconstructed object points when coherent light is used as reference light. Extant studies proposed the pixel separation method [29], [30] and the random pixel separation method [31] to reduce speckle noise. In 2015, a study improved the image quality of electroholography by applying a spatiotemporal division multiplexing method [32]. However, the spatiotemporal division multiplexing method requires the SLM to play back the computer-generated hologram at a high speed.

The present study involved proposing a method of high-speed reconstruction of a computer-generated hologram by using a digital micromirror device (DMD) [33] for high-definition spatiotemporal division multiplexing electroholography.

The rest of this paper is structured as follows. Section 2 describes a binary CGH computation. The proposed high-speed holographic movie playback using the DMD is outlined in Sect. 3. The experimental results are presented and discussed in Sect. 4. The conclusions are presented in Sect. 5 .

\section{Binary Computer-Generated Hologram Computa- tion}

A simple algorithm was used to calculate the binary CGH obtained from a 3-D object expressed by a point cloud for in-line holography. With respect to a 3-D object composed of $N$ points, the light intensity of a point on the hologram is calculated by using an equation [15] as follows:

$$
I\left(x_{h}, y_{h}, 0\right)=\sum_{j=1}^{N} A_{j} \cos \left\{\frac{\pi}{\lambda z_{j}}\left[\left(x_{h}-x_{j}\right)^{2}+\left(y_{h}-y_{j}\right)^{2}\right]\right\},
$$

where $I\left(x_{h}, y_{h}, 0\right)$ denotes the light intensity of the point $\left(x_{h}, y_{h}, 0\right)$ on the hologram, $\left(x_{j}, y_{j}, z_{j}\right)$ denotes the coordinate of the $j$-th point on the 3 -D object, $A_{j}$ denotes the amplitude of the object point, and $\lambda$ denotes the wavelength of the reference light. Equation (1) is obtained by using the Fresnel approximation. In this study, the parameter $A_{j}$ of all object points is set as 1.0. Following the calculation of Eq. (1), the light intensity of each point on the hologram is binarized by 
using a threshold value of zero [34].

\section{High-Speed Holographic Movie Playback by Using a Digital Micromirror Device}

Figure 1 shows an outline of the spatiotemporal division multiplexing method [32] when the number of spatial divisions corresponds to six. In Fig. 1, the N-th frame (Frame $\mathrm{N}$ ) of the original 3-D movie is divided into subframes that are labeled as "DivN-1" to "DivN-6." In the spatial division of "Frame N," the serial number of each object point on the original 3-D object is used. Figure 2 illustrates the outline of the spatial division technique [19] used in the study when the number of spatial divisions corresponds to three. The spatial division technique proceeds as follows:

Step 1. All object points on the original 3-D object are serially numbered as $\mathrm{P} 1, \mathrm{P} 2$, and $\mathrm{P} 3, \ldots$.

Step 2. In the original data file shown in Fig. 2, the coordinate data of the object points are listed in ascending order of the serial object point number.

Step 3. As shown in Fig. 2, the listed coordinate data in the original data file are divided into three sub-files (Files $1-3$ ) in ascending order of the serial object point number.

It should be noted that all object points on the original 3-D object are serially numbered as P1, P2, and P3, . at Step 1 such that the object points stored in each sub-file are evenly distributed over the entire area of the original 3-D object.

All CGHs obtained from all sub-frames of Frame $\mathrm{N}$ are individually displayed on a SLM. The total number of subframes is proportional to the number of spatial divisions. Therefore, the spatiotemporal division multiplexing method requires a high-refresh-rate SLM to play all sub-frames at a high-speed.

The DMD displays color movies at a high refresh rate. The reproduction of color images using DMD is shown in the upper time-chart of Fig. 3. In each frame, the DMD reproduces a color image by sequentially displaying the corresponding RGB images as shown in the upper time-chart of Fig. 3. Specifically, $R, G$, and $B$ indicate red, green, and blue colored images, respectively. Additionally, $T_{R}, T_{G}$, and $T_{B}$ correspond to the display time periods of the red, green, and blue images, respectively. A single-frame refresh period is equally divided into three display time periods (i.e., $T_{R}, T_{G}$, and $T_{B}$ ). The RGB images are displayed at a refresh rate of $120 \mathrm{~Hz}$ [33]. Thus, the sum of $T_{R}, T_{G}$, and $T_{B}$ corresponds to $1 / 120 \mathrm{~Hz} \approx 8.3 \mathrm{~ms}$. The color image projected by the DMD depends on the color of the projection light. Therefore, a color image is faithfully projected on the screen when the color-switching of the projection light is synchronized with the display of the suitable RGB image on the DMD.

As shown in the lower time-chart of Fig. 3, the CGHs corresponding to the sub-frames "DivN-1," "DivN-2," and "DivN-3" are converted to red, green, and blue colored CGHs, respectively. The colored CGHs with an 8-bit depth form a 24-bit colored image. The color image is output from

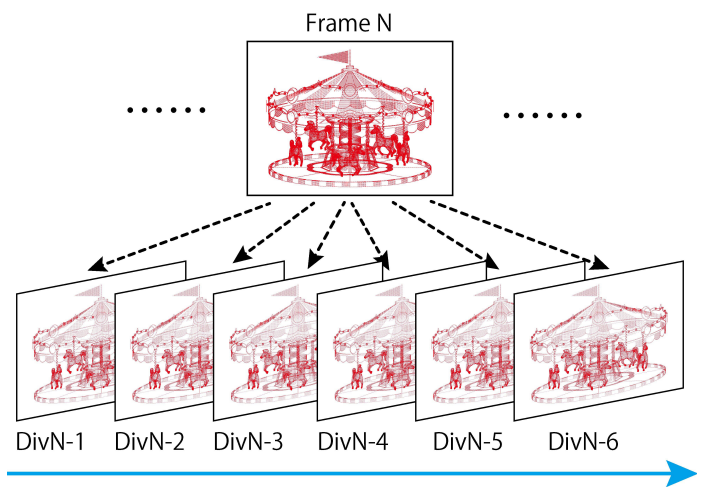

Fig. 1 High-definition spatiotemporal division multiplexing electroholography.
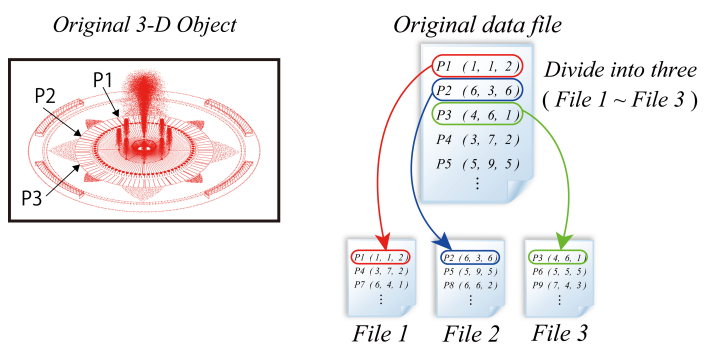

Fig. 2 Outline of the spatial division technique.

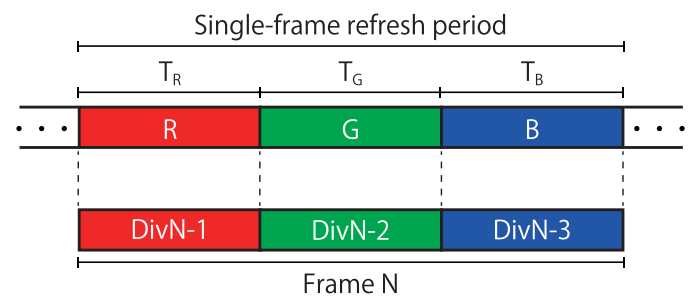

Fig. 3 Time chart of the 3-D movie reproduction using the RGB-mode method.

the GPU and fed into the DMD system via the DisplayPort. The DMD system automatically displays three CGHs corresponding to the sub-frames, namely "DivN-1," "Div-N-2," and "DivN-3" in sequence as shown in the lower time-chart of Fig. 3. Monochrome light is used as the reference light because the 3-D movie reconstructed through electroholography (as shown in Fig. 1) is monochrome. The frame rate of this method is termed as the "RGB-mode method" and corresponds to $120 \mathrm{~Hz} \times 3$ (color $)=360 \mathrm{~Hz}$.

Furthermore, it is proposed that two frames of a monochromatic 3-D movie are assigned to each color image $(R, G$, and $B)$ of the conventional DMD reproduction as shown in Fig. 4. In the proposed method, binary CGH [34] and monochrome reference light are used. Figure 5 shows the lighting time of three red images with gradation values corresponding to 127,128 , and 255 . A DMD chip has millions of microscopic mirrors arranged in a rectangular array that correspond to the pixels in the image to be displayed. The mirrors are individually rotated to an "ON" or "OFF" 


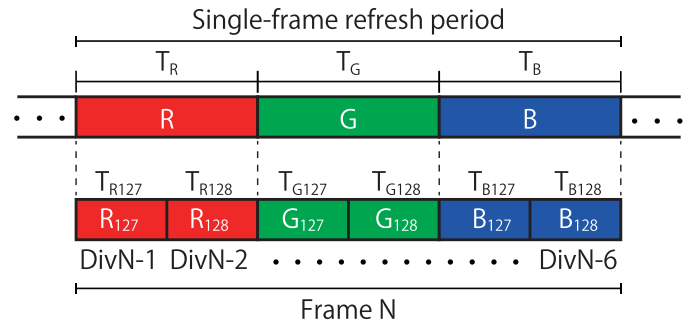

Fig. 4 Time chart of 3-D movie reproduction by using the proposed method

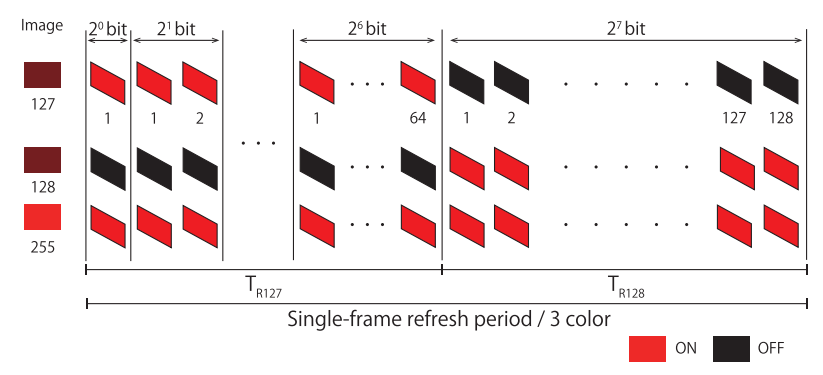

Fig. 5 Binary PWM-sequence pattern.

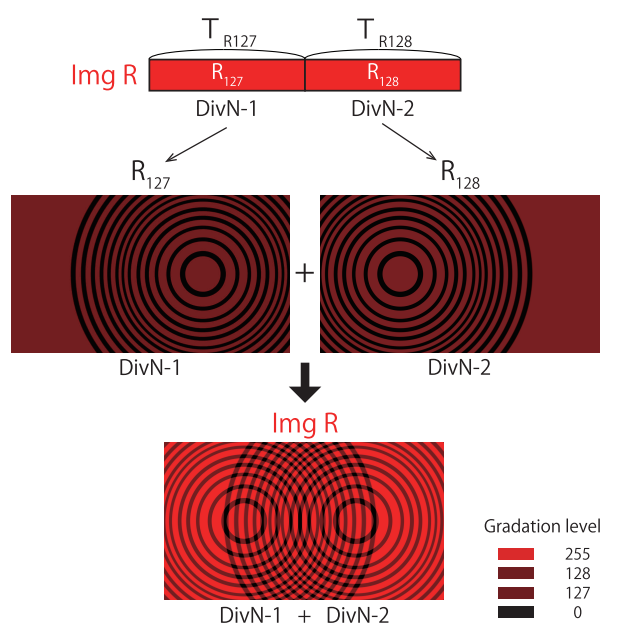

Fig. 6 The method of incorporating two CGHs into a red-colored image.

state very quickly. In the "ON" state, the light from the light source is reflected to the screen, and this makes the pixel appear bright to a viewer. In the "OFF" state, the light is directed to a light absorber material, and this essentially makes the pixel appear dark on the screen. Therefore, the DMD expresses the gradation of each color image by using binary pulse width modulation (PWM) as shown in Fig. 5 [35]. In Fig. 5, three red images are provided with gradation values proportional to the percentage of time when the mirrors are in the "ON" state during one-third of a single-frame refresh period. The lighting time of the red image with the gradation value of 128 is nearly equal to that of the image with the gradation value corresponding to 127 . The conventional binary CGH is illustrated in bicolor. The binary CGHs "R127" and "R128" are drawn in black and the red colors with gradation values corresponding to 127 and 128, respectively, as shown in Fig. 6. In order to display the binary CGHs, the mirrors

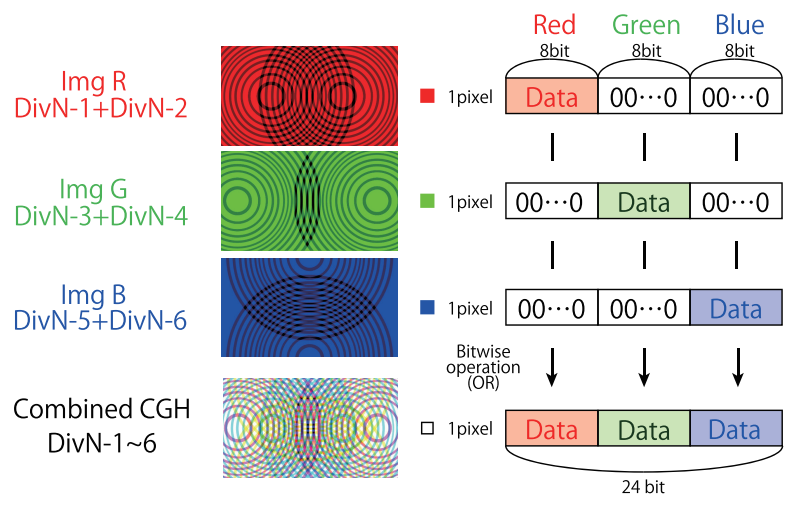

Fig. 7 The method of incorporating six CGHs into a color image.

corresponding to the pixels on the black and red areas of the binary CGHs correspond to the "OFF" state and "ON" state, respectively. In Fig. 6, the binary CGHs "R127" and "R128" are assigned to the "Frame N" sub-frames "DivN1" and "DivN-2," respectively (Fig. 4). A RGB color image in the DMD is expressed in 24 bits. However, the gradation of each color image $(R, G$, and $B)$ shown in the upper time-chart of Fig. 4 is expressed in 8 bits. Therefore, the two CGH images "R127" and "R128" are combined into a single image "Img R" as shown in Fig. 6. All sub-frames from "DivN-1" to "DivN-6" as shown in Fig. 1 are combined into a color image as shown in Fig. 7. Finally, as shown in Fig. 1, "Frame N" is displayed at the frame rate of $120 \mathrm{~Hz}$ when the number of spatial divisions corresponds to six because each sub-frame is displayed at a frame rate of $720 \mathrm{~Hz}(120 \mathrm{~Hz} \times 3($ color $) \times 2($ frame $))$.

\section{Results and Discussion}

The proposed gradation method is evaluated by using the optical setup shown in Fig. 8. In the setup, a laser light with a wavelength of $532 \mathrm{~nm}$ is use as the reference light, a DLP LightCrafter 6500 (Texas Instruments, Micro-mirror pixel pitch: $7.6 \mu \mathrm{m}$, Micromirror array size: $1920 \times 1080$ ) [33] is used as the DMD system, and a digital camera (Canon EOS 6D) with a 35-mm CMOS image sensor is also used. In the CGH computation, a PC equipped with an Intel Core i7 4770 processor (Clock Speed: $3.4 \mathrm{GHz}$, quad-core) a Linux (CentOS 7.1) operating system, a NVIDIA GeForce GTX TITAN X GPU, and a CUDA 7.0 software-development kit is used for GPU programming. The distance between the 3 -D object and the CGH corresponds to $1.0 \mathrm{~m}$. The refresh rate of DLP LightCrafter 6500 corresponds to $120 \mathrm{~Hz}$ when the DMD system connects to the GPU via the DisplayPort [33]. In the study, the DisplayPort is used to connect to the DMD system. In the RGB-mode, three subframes, namely "DivN-1," "DivN-2," and "DivN-3" are displayed for a single-frame refresh period $(1 / 120 \mathrm{~Hz} \approx 8.3 \mathrm{~ms})$ as shown in Fig. 3. The frame rate of RGB mode corresponds to $120 \mathrm{~Hz} \times 3$ (color) $=360 \mathrm{~Hz}$. In the proposed method, six subframes numbered "DivN-1" to "DivN-6" are displayed for a single-frame refresh period $(1 / 120 \mathrm{~Hz} \approx 8.3 \mathrm{~ms})$ as 


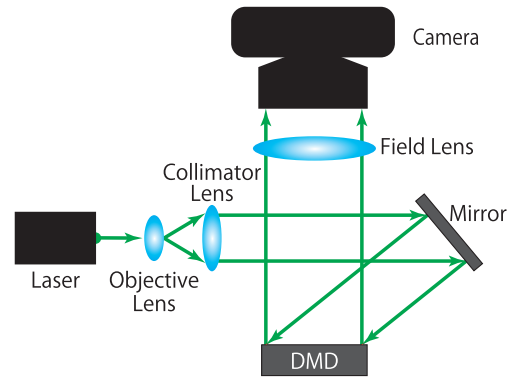

Fig. 8 Optical setup
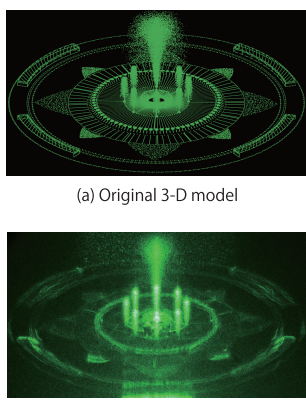

(c) 3-division

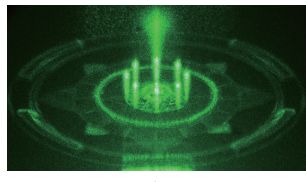

(e) 9-division (a) Original 3-D model

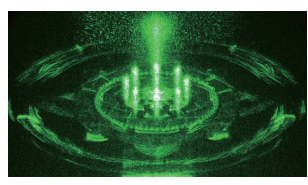

(b) No-division

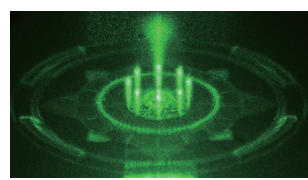

(d) 6-division

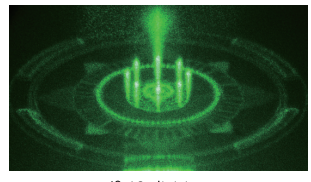

(f) 12-division
Fig.9 Still images produced through spatiotemporal division multiplexing electroholography with a DMD.

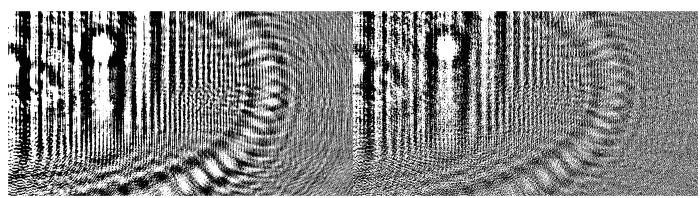

(a) No-division

(b) 12-division

Fig. 10 The CGHs are generated by using no-division and 12-division.

shown in Fig. 4. The frame rate of proposed method corresponds to $120 \mathrm{~Hz} \times 3($ color $) \times 2($ frame $)=720 \mathrm{~Hz}$.

The reconstructed still image of the 3-D model that is comprised of 912,462 points as shown in Fig. 9 (a) is evaluated. Figure 9 shows that the reconstructed still 3-D image obtained through spatiotemporal division multiplexing electroholography is clear when the number of divisions corresponds to twelve. Therefore, twelve-division spatiotemporal division multiplexing electroholography is adapted for the optical experiment by using the DMD. Figure 10 (a) and (b) show the CGHs of no-division and twelve-division, respectively. The CGH shown in Fig. 10 (b) correspond to fine interference fringes when compared with the CGH shown in Fig. 10 (a).

Figure 11 shows the snapshots of the reconstructed 3$\mathrm{D}$ movie that are played back at frame-rates corresponding to $60-\mathrm{Hz}, 120-\mathrm{Hz}, 360-\mathrm{Hz}$, and $720-\mathrm{Hz}$. The reconstructed

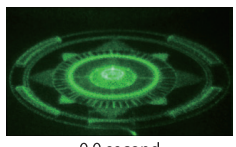

0.0 second

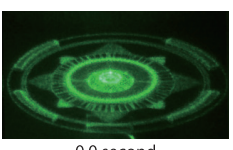

0.0 second

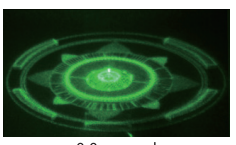

0.0 second

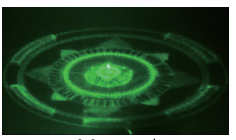

0.0 second
3.08 seconds
(d) Proposed method $(720 \mathrm{~Hz})$

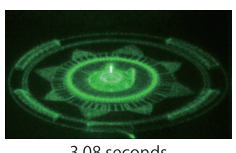

3.08 seconds (a) Conventional mode $(60 \mathrm{~Hz})$

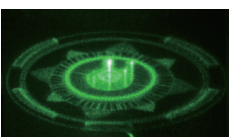

3.08 seconds (b) Conventional video mode $(120 \mathrm{~Hz})$

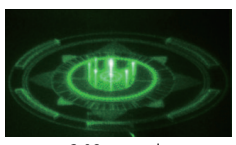

3.08 seconds (c) RGB-mode method $(360 \mathrm{~Hz}$

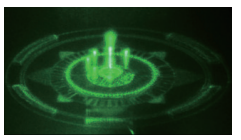

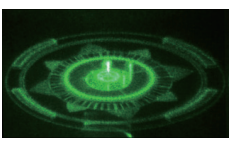

6.15 seconds

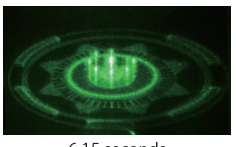

6.15 seconds

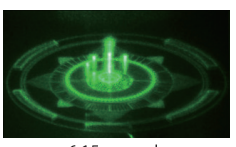

6.15 seconds

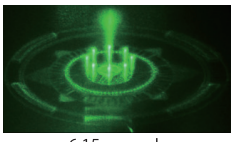

6.15 seconds
Fig. 11 Snapshots of the reconstructed 3-D movie.

Table 1 The playback time of the reconstructed 3-D movie.

\begin{tabular}{lc}
\hline & playback time (ms) \\
\hline Conventional method & 80.34 \\
Video mode & 40.13 \\
RGB mode & 13.54 \\
Proposed method & 6.85 \\
\hline
\end{tabular}

Table 2 The playback speed of the reconstructed 3-D movie comprised of 4,788 frames.

\begin{tabular}{lc}
\hline & playback speed (ms/frame) \\
\hline Conventional method & 16.78 \\
Video mode & 8.38 \\
RGB mode & 2.83 \\
Proposed method & 1.43 \\
\hline
\end{tabular}

3-D movie is comprised of 4,788 frames. The reconstructed $3-\mathrm{D}$ movie is played back at frame-rates of $120 \mathrm{~Hz}, 360 \mathrm{~Hz}$, and $720 \mathrm{~Hz}$ by using the video mode [33], the RGB mode, and the proposed method, respectively. Table 1 and Table 2 show the playback time and the speed of the reconstructed 3 -D movie, respectively. Therefore, the proposed method is used to realize high-speed movie playback.

\section{Conclusion}

In this study, a method was developed for high-speed holographic movie playback by using a DMD. It is extremely easy to implement the proposed method because high-speed holographic movie playback is executed through color images obtained from six binary CGHs of the 3-D movie. The proposed method was further applied to reconstruct a highdefinition 3-D movie of a 3-D object that is approximately comprised of 900,000 points by using spatiotemporal division multiplexing electroholography with the DMD. 


\section{Acknowledgments}

This work was partially supported by the Japan Society for the Promotion of Science through a Grant-in-Aid for Scientific Research (C) 15K00153 and a Grant-in-Aid for Scientific Research (A), 25240015.

\section{References}

[1] D. Gabor, "A new microscopic principle," Nature, vol.161, no.4098, pp.777-778, 1948.

[2] G. Tricoles, "Computer generated holograms: an historical review," Appl. Opt., vol.26, no.20, pp.4351-4360, 1987.

[3] S.A. Benton and J.V.M. Bove, Holographic Imaging, Wiley, 2008.

[4] T. Kakue, T. Nishitsuji, T. Kawashima, K. Suzuki, T. Shimobaba, and T. Ito, "Aerial projection of three-dimensional motion pictures by electro-holography and parabolic mirrors," Sci. Rep., vol.5, 2015.

[5] T. Inoue and Y. Takaki, "Table screen 360-degree holographic display using circular viewing-zone scanning," Opt. Express, vol.23, no.5, pp.6533-6542, 2015.

[6] Y. Lim, K. Hong, H. Kim, H.-E. Kim, E.-Y. Chang, S. Lee, T. Kim, J. Nam, H.-G. Choo, J. Kim, and J. Hahn, "360-degree tabletop electronic holographic display," Opt. Express, vol.24, no.22, pp.24999-25009, 2016.

[7] T. Shimobaba, T. Kakue, and T. Ito, "Review of fast algorithms and hardware implementations on computer holography," IEEE Trans. Industrial Informatics, vol.12, no.4, pp.1611-1622, 2016.

[8] T. Shimobaba, N. Masuda, and T. Ito, "Simple and fast calculation algorithm for computer-generated hologram with wavefront recording plane," Opt. Lett., vol.34, no.20, pp.3133-3135, 2009.

[9] S.-C. Kim and E.-S. Kim, "Effective generation of digital holograms of three-dimensional objects using a novel look-up table method," Appl. Opt., vol.47, no.19, pp.D55-D62, 2008.

[10] T. Nishitsuji, T. Shimobaba, T. Kakue, and T. Ito, "Fast calculation of computer-generated hologram using run-length encoding based recurrence relation," Opt. Express, vol.23, no.8, pp.9852-9857, 2015.

[11] T. Nishitsuji, T. Shimobaba, T. Kakue, D. Arai, and T. Ito, "Simple and fast cosine approximation method for computer-generated hologram calculation," Opt. Express, vol.23, no.25, pp.32465-32470, 2015.

[12] P. Su, W. Cao, J. Ma, B. Cheng, X. Liang, L. Cao, and G. Jin, "Fast Computer-Generated Hologram Generation Method for Three-Dimensional Point Cloud Model," J. Display Technol., vol.12, no.12, pp.1688-1694, 2016

[13] T. Shimobaba and T. Ito, "Fast generation of computer-generated holograms using wavelet shrinkage," Opt. Express, vol.25, no.1, pp.77-87, 2017.

[14] K. Matsushima and S. Nakahara, "Extremely high-definition fullparallax computer-generated hologram created by the polygon-based method," Appl. Opt., vol.48, no.34, pp.H54-H63, 2009.

[15] N. Masuda, T. Ito, T. Tanaka, A. Shiraki, and T. Sugie, "Computer generated holography using a graphics processing unit," Opt. Express, vol.14, no.2, pp.603-608, 2006.

[16] Y. Pan, X. Xu, S. Solanki, X. Liang, R.B.A. Tanjung, C. Tan, and T.-C. Chong, "Fast CGH computation using S-LUT on GPU," Opt. Express, vol.17, no.21, pp.18543-18555, 2009.

[17] N. Takada, T. Shimobaba, H. Nakayama, A. Shiraki, N. Okada, M. Oikawa, N. Masuda, and T. Ito, "Fast high-resolution computergenerated hologram computation using multiple graphics processing unit cluster system," Appl. Opt., vol.51, no.30, pp.7303-7307, 2012.

[18] B.J. Jackin, H. Miyata, T. Ohkawa, K. Ootsu, T. Yokota, Y. Hayasaki, T. Yatagai, and T. Baba, "Distributed calculation method for large-pixel-number holograms by decomposition of object and hologram planes," Opt. Lett., vol.39, no.24, pp.6867-6870, 2014

[19] H. Niwase, N. Takada, H. Araki, H. Nakayama, A. Sugiyama, T. Kakue, T. Shimobaba, and T. Ito, "Real-time spatiotemporal division multiplexing electroholography with a single graphics processing unit utilizing movie features," Opt. Express, vol.22, no.23, pp.28052-28057, 2014.

[20] P. Tsang, W.-K. Cheung, T.-C. Poon, and C. Zhou, "Holographic video at 40 frames per second for 4-million object points," Opt. Express, vol.19, no.16, pp.15205-15211, 2011.

[21] J. Weng, T. Shimobaba, N. Okada, H. Nakayama, M. Oikawa, N. Masuda, and T. Ito, "Generation of real-time large computer generated hologram using wavefront recording method," Opt. Express, vol.20, no.4, pp.4018-4023, 2012.

[22] F. Yaraş, H. Kang, and L. Onural, "Real-time phase-only color holographic video display system using LED illumination," Appl. Opt., vol.48, no.34, pp.H48-H53, 2009.

[23] H. Nakayama, N. Takada, Y. Ichihashi, S. Awazu, T. Shimobaba, N. Masuda, and T. Ito, "Real-time color electroholography using multiple graphics processing units and multiple high-definition liquid-crystal display panels," Appl. Opt., vol.49, no.31, pp.5993-5996, 2010.

[24] J. Song, C. Kim, H. Park, and J.-I. Park, "Fast generation of a highquality computer-generated hologram using a scalable and flexible PC cluster," Appl. Opt., vol.55, no.13, pp.3681-3688, 2016.

[25] H. Niwase, N. Takada, H. Araki, Y. Maeda, M. Fujiwara, H. Nakayama, T. Kakue, T. Shimobaba, and T. Ito, "Real-time electroholography using a multiple-graphics processing unit cluster system with a single spatial light modulator and the InfiniBand network," Opt. Eng., vol.55, no.9, 2016.

[26] H. Sasaki, K. Yamamoto, K. Wakunami, Y. Ichihashi, R. Oi, and T. Senoh, "Large size three-dimensional video by electronic holography using multiple spatial light modulators," Sci. Rep., vol.4, 2014.

[27] H. Araki, N. Takada, H. Niwase, S. Ikawa, M. Fujiwara, H. Nakayama, T. Kakue, T. Shimobaba, and T. Ito, "Real-time timedivision color electroholography using a single GPU and a USB module for synchronizing reference light," Appl. Opt., vol.54, no.34, pp.10029-10034, 2015.

[28] T. Horikoshi, T. Akimoto, K. Higuchi, and N. Sonehara, "Time sharing displaying method for displaying Computer Generated Hologram,” ITE Technical Report, vol.22, no.43, pp.17-21, 1998.

[29] Y. Takaki and M. Yokouchi, "Speckle-free and grayscale hologram reconstruction using time-multiplexing technique," Opt. Express, vol.19, no.8, pp.7567-7579, 2011.

[30] M. Makowski, "Minimized speckle noise in lens-less holographic projection by pixel separation," Opt. Express, vol.21, no.24, pp.29205-29216, 2013.

[31] Y. Mori, T. Fukuoka, and T. Nomura, "Speckle reduction in holographic projection by random pixel separation with time multiplexing," Appl. Opt., vol.53, no.35, pp.8182-8188, 2014.

[32] Y. Maeda, N. Takada, H. Niwase, H. Araki, M. Fujiwara, S. Ikawa, H. Nakayama, T. Kakue, T. Shimobaba, and T. Ito, "Image Quality Improvement of Electroholography by Using Spatiotemporal Division Method," Proc. IDW'15, pp.908-909, 2015.

[33] TI DLP LightCrafter 6500 and 9000 EVM User's Guide, Available: http://www.ti.com/lit/ug/dlpu028c/dlpu028c.pdf

[34] W.-H. Lee, "Binary computer-generated holograms," Appl. Opt., vol.18, no.21, pp.3661-3669, 1979.

[35] D. Dudley, W.M. Duncan, and J. Saughter, "Emerging digital micromirror device (DMD) applications," Proc. SPIE, vol.4985, 2003. 


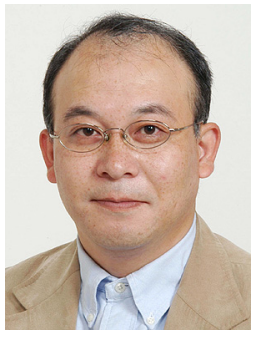

Naoki Takada received his B.E., M.E., and Ph.D degrees in electrical engineering from Gunma University, Japan in 1994, 1996, and 2000, respectively. From 1996 to 2001, he was a research associate at Oyama National College of Technology, Japan. From 2001 to 2005, he was a research scientist at the High-Performance Biocomputing Research Team, Bioinformatics Group, Genomic Science Center (GSC), RIKEN, Japan. He was a lecturer from 2005 to 2010 and an associate professor from 2010 to 2012, at Shohoku College, Japan. Since 2012, he has been an associate professor at Science Department, Natural Sciences Cluster, Research and Education Faculty, Kochi University, Japan. His research interests are highperformance computing and electroholography. He is a member of ACES, IEICE, and OSA.

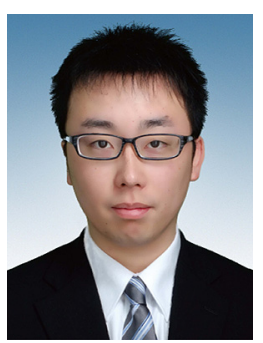

Masato Fujiwara received his B.S. and M.S. degrees in information science from Kochi University, Japan, in 2015, and 2017, respectively. His research interests are gradation representation of electroholography and high-speed 3-D electroholographic movie playback using a DMD.

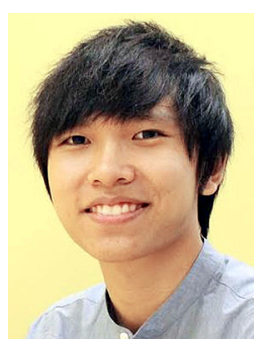

Chun Wei Ooi graduated from Kisarazu National College of Technology, Japan, in 2015. He received his B.S. degree in information science from Kochi University, Japan, in 2017. Currently, he is a graduate student at Graduate School of Library, Information and Media Studies, University of Tsukuba, Japan. His research interest is high-speed 3-D electroholographic movie playback using a DMD.

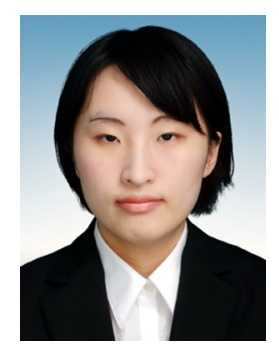

Yuki Maeda received her B.S. and M.S. degrees in information science from Kochi University, Japan, in 2015, and 2017, respectively. Her research interest is image quality improvement of electroholography.

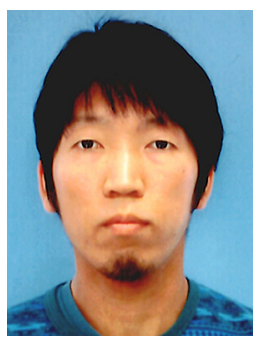

Hirotaka Nakayama received his B.E. and M.E. degrees in informatics and imaging and Ph.D. degree in electrical engineering from Chiba University, Japan, in 2002, 2004, and 2011, respectively. He is a research expert at the Center for Computational Astrophysics, National Astronomical Observatory of Japan. His research interests are computer graphics and image processing. He is a member of IEICE.

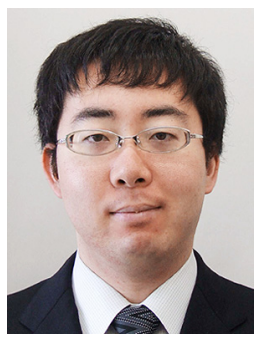

Takashi Kakue received his B.E., M.E., and Ph.D. degrees in electronics and information science from Kyoto Institute of Technology, Japan, in 2006 and 2008, and 2012, respectively. Since 2012, he has been an assistant professor at the Graduate School of Engineering, Chiba University, Japan. His research interests are holography, digital holography, computer holography, holographic interferometry, 3D imaging, highspeed imaging, and ultrafast optics. He is a member of IEEE, OSA, OSJ, and SPIE.

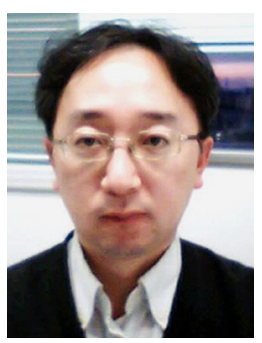

Tomoyoshi Shimobaba received his B.E. and M.E. degrees in electrical engineering from Gunma University, Japan, in 1997 and 1999, respectively. And he received a Ph.D. degree from Chiba University, Japan in 2002. From 2002 to 2005 , he was a special postdoctoral researcher at RIKEN. From 2005 to 2009, he was an associate professor at the Graduate School of Science and Engineering, Yamagata University. Since 2009, he has been an associate professor at the Graduate School of Engineering, Chiba University. His research interests are computer holography and its applications. He is a member of ITE, IEICE, OSA, OSJ, and SPIE.

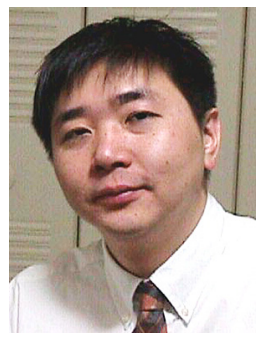

Tomoyoshi Ito received his B.S. degree in pure and applied Sciences, M.S. and Ph.D. degrees in earth science and astronomy from the University of Tokyo, Japan, in 1989, 1991, and 1994, respectively. He was a research associate from 1992 to 1994 and an associate professor from 1994 to 1999 at Gunma University, Japan. From 1999 to 2004, he was an associate professor at the Graduate School of Engineering, Chiba University, Japan, and since 2004, he has been a professor. His research interests are highperformance computing and its applications, such as electronic holography for 3D TV. He is a member of ACM, ASJ, ITE, IEICE, IPSJ, and OSA. 